\title{
ANÁlISIS DE LA NUPCIALIDAD LEGAL POR GENERACIONES EN MÉXICO, 1922-1969
}

\author{
Alain Jourdain * \\ Julieta QuilodráN DE AGUirRe \\ El Colegio de México
}

\section{INTRODUCCIÓN}

EL PRESENTE ESTUdro constituye un ensayo de análisis demográfico so. bre la evolución de la nupcialidad por generaciones en México durante el período comprendido entre el final de la revolución y el año de 1969.

Por "análisis demográfico" se entenderá el conjunto de métodos que permiten poner en evidencia la influencia de cada una de las variables demográficas sobre las variaciones de un fenómeno dado, en este caso la nupcialidad. A partir de las variaciones del número absoluto de ma. trimonios se analizará el comportamiento de algunas variables tales como la edad promedio al matrimonio o la intensidad del mismo.

Cualquier fenómeno demográfico se puede analizar "transversalmente" si se utiliza información a un año dado o, en forma "longitudinal" si el interés se centra en la información relativa a una generación o grupo de generaciones.

En el caso de un fenómeno como la nupcialidad, en que el número de matrimonios celebrados en años consecutivos puede variar en forma importante, el análisis transversal no es adecuado porque puede revelar una imagen falsa de las tendencias que está siguiendo la nupcialidad. $\mathrm{Si}$ el análisis se efectúa en un momento en que se está produciendo una postergación masiva de los matrimonios por causas coyunturales, la conclusión puede ser que la nupcialidad está en descenso. La conclusión contraria podría obtenerse si el análisis se efectúa en un momento de recuperación de la nupcialidad.

Ahora bien, si el análisis se realiza en el seno de las generaciones, los efectos que tienen las causas de tipo coyuntural sobre la nupcialidad se ven atenuadas. Los efectos de postergación y recuperación llegan a compensarse casi totalmente a través de la historia de un mismo grupo de generaciones, de modo que lo que revelan las variaciones entre generaciones en realidad son cambios de tendencias a largo plazo. Por esta razón, el enfoque más adecuado es el generacional, dados los

* Trabajo elaborado durante su estancia en el Centro de Estudios Económicos y Demográficos como becario honorario. 
propósitos de estudiar los cambios duraderos que ha sufrido la nupcialidad durante el período considerado.

Fuente de datos. El censo es la fuente de información más completa para efectuar análisis de la nupcialidad por generaciones en la medida que proporciona datos sobre los diferentes tipos de estado civil. Para seguir la evolución de la nupcialidad por generaciones es necesario disponer de la distribución de la población por grupos de edad y por estado civil, por lo menos de tres censos consecutivos. En México solamente los censos de 1960 y 1970, y, en cierto modo, el de 1930, proporcionan la información mínima requerida. Lo poco adecuado de la clasificación por estado civil y grupos de edad en el censo de 1940 y su inexistencia en 1950, imposibilita la reconstrucción de los grupos de generaciones y por lo tanto cualquier estudio de tipo longitudinal de la nupcialidad a través de esta fuente de información.

La alternativa común al análisis longitudinal, sería un estudio de tipo transversal en las fechas censales. Sin embargo, este enfoque no es el adecuado para el caso de México, ya que la nupcialidad y la estructura del estado civil de la población han experimentado fuertes cambios, especialmente a partir de $1930 .{ }^{1}$

Esta situación obliga a utilizar en el análisis la información de las estadísticas contínuas del Registro Civil. Esta fuente de información limita el estudio de la evolución de la nupcialidad, debido a que los datos sólo posibilitan el examen de la unión legal (civil) en su conjunto, sin que se puedan distinguir las uniones de primer orden de las del resto, ${ }^{2}$ por carecer de la clasificación de los matrimonios según el estado civil anterior de los contrayentes.

Sin embargo, en la información publicada en forma especial en 1939 por la Dirección General de Estadística, ${ }^{3}$ se observa que el porciento de personas que contrajeron matrimonio de segundo orden o posterior en ese año fue muy bajo (véase el cuadro 1). Lo que se desconoce por completo para México es la distribución de las segundas nupcias en adelante según la edad de los contrayentes, lo cual implicó clasificar los matrimonios según el estado civil anterior de los contrayentes. La existencia de este tipo de datos hubiera permitido el análisis de la formación de las primeras uniones que son las que presentan mayor interés.

A pesar de este inconveniente se considera importante efectuar un onálisis de tipo longitudinal por ser la única forma de captar las variaciones reales de la nupcialidad por edad a través del tiempo, aún teniendo en cuenta que el examen sólo indicará, en forma gruesa, la evolución de este fenómeno.

1 Véase J. Quilodrán, "Evolución de la nupcialidad en México 1900-1970". Demografía y Economía, El Colegio de México, Vol. VIII, Núm. 1, 1974, pp. 34-49.

2 Se entiende por unión de primer orden a la primera celebrada legalmente.

3 Cf. Dirección General de Estadística, s.I.c. Anuario Estadístico de los Estados Unidos Mexicanos. 
Cuadro 1

México: Distribución de los MATRIMonios totales Y DE PRimer ORDEN EN 1939

\begin{tabular}{lrr}
\hline & Hombros & Majoros \\
\hline $\begin{array}{l}\text { Nimero de } \\
\text { primeroa matrimonios (1) }\end{array}$ & 125131 & 129887 \\
$\begin{array}{l}\text { Nimero total do } \\
\text { matrimonios (2) }\end{array}$ & 133746 & 133746 \\
$\begin{array}{l}\text { Poroionto do } \\
\text { primeros matrimonios (1)/(2) }\end{array}$ & 93.6 & 97.1 \\
\hline
\end{tabular}

Fuente: Dirección General de Estadística, Secretaría de Industria y Comercio, Anuario Estadístico, 1939.

\section{MEdición DE LA NUPCiALidAd CIVIL EN LAS GENERACIONES}

Los fenómenos demográficos pueden clasificarse, con respecto al número de veces que pueden ocurrirle a una misma persona, en "renovables" y "no renovables". La mortalidad es por definición un fenómeno que puede ser calificado de "no renovable" ya que un individuo no puede morirse más que una vez, mientras la fecundidad, representada por el número de hijos por mujer, constituye un acontecimiento de tipo renovable puesto que puede ocurrirle varias veces a una misma mujer.

En la práctica las tasas constituyen uno de los indicadores usados con mayor frecuencia para medir los acontecimientos demográficos. Estas pueden ser definidas, de una manera muy general, como la relación entre el número de acontecimientos ocurridos durante un período dado y el efectivo de la población que está en condiciones de vivir el acontecimiento durante el mismo período. Sin embargo, el carácter renovable o no renovable del acontecimiento que se está considerando hará cambiar la población de referencia que se utiliza en el cálculo de la tasa respectiva. Por ejemplo, si se considera la nupcialidad de primer orden (nupcialidad de los solteros) que es un acontecimiento no renovable, la población de referencia que se utilizará en el cálculo de la tasa será la población de solteros, por ser éstos los únicos que están en condiciones de contraer un primer matrimonio. En cambio, si se trata de calcular la tasa de nupcialidad sin distinguir orden de unión, la población de referencia estará compuesta por el total de población en edad de contraer matrimonio, ya que en este caso la nupcialidad pasa a constituir un fenómeno renovable y todos, solteros o casados, pueden contraer nupcias. En el primer caso, la tasa es conocida con el nombre de tasa de primera categoría y en el segundo caso como tasa de segurida categoría. ${ }^{4}$

Con base en el criterio de fenómenos no renovables y renovables

4 R. Pressat, L'analyse démographique, $2^{\mathrm{a}}$ edición, París, Presses Universitaires de France, 1969, p. 37. 
se asocia a los primeros con las "tablas" (de mortalidad, de nupcialidad de solteros, de fecundidad de primer orden, etc.) y a los segundos con lo que Pressat ha dado en denominar "cuadros". 5

Por las mismas razones, la interpretación de los datos contenidos en uno y otro es diferente. En el caso de una "tabla", el número de acontecimientos es igual al número de individuos que sufren dicho acontecimiento por el hecho de que es "no renovable" y de que cada persona sólo lo puede experimentar una vez. Cuando se trata de un "cuadro", el acontecimiento es "renovable", es decir, puede repetirse y por lo tanto el número de ellos suele ser mayor que el número de personas que lo sufren. En ambos casos el número de acontecimientos refleja la "intensidad" del fenómeno en la población en la cual se está midiendo.

Los estudios que se realizan sobre nupcialidad, generalmente centran su atención sobre la formación de las primeras uniones, dadas las implicaciones que esta formación tiene sobre otros fenómenos demográficos y sociales. Por desgracia, las características de los datos disponibles señaladas anteriormente han impedido separar las primeras uniones del conjunto de uniones registradas y conducir el análisis sobre ellas.

Ante esta dificultad, se adoptó un procedimiento especial para el tratamiento de la información sobre nupcialidad, diferente al habitual y adecuado a las características de la información. La innovación consiste fundamentalmente en que se considera la nupcialidad como un fenómeno "renovable" 6 y bajo esta condición, se aplicaron las formas de análisis apropiadas a este tipo de fenómenos.

Esto significa que el número de matrimonios en una generación se estudia de manera similar a la forma en que se analiza el número de niños nacidos vivos producto de una generación de madres que no ha experimentado ni mortalidad ni migración. Es decir, se reemplaza el concepto número de hijos nacidos vivos por mujer, en ausencia de mortalidad y migración, por el número de matrimonios por persona en ausencia de mortalidad y migración.?

\section{Cálculo de las tasas de nupcialidad de segunda categoría}

La superficie sombreada del diagrama de Lexis que aparece en la gráfica 1, representa los datos sobre nupcialidad tal como aparecen en los Anuarios Estadísticos: número de matrimonios registrados durante el año $z$ para el grupo de edades en años cumplidos $(x, x+4)$; es decir, para un grupo quinquenal de edades para el año civil.

5 "Cuadro" es la traducción literal del término utilizado por Pressat en francés, véase R. Pressat, op. cit.

6 L. Henry, "Analyse des phenomènes demographiques par cohortes", Population, 1966, p. 466.

7 En el apéndice metodológico aparece la demostración de la relación existente entre las tasas de nupcialidad de segunda categoría y el número medio de matrimonios por persona, en ausencia de mortalidad y migración. 
Los datos anuales sobre matrimonios civiles clasificados por grupos quinquenales de edad, publicados desde 1922 hasta 1969, permiten la obtención de una serie de tasas de nupcialidad de segunda categoría a partir de las cuales se constuyeron "cuadros de nupcialidad por generaciones" en los que se observan las características fundamentales del fenómeno nupcialidad que serán analizadas.

El procedimiento seguido para el cálculo de las tasas (véase la gráfica 1) consistió en:

a) Sumar los matrimonios registrados de personas de edad ( $x$, $x+4)$ para 5 años consecutivos $(z, z+4)$. Esta suma se expresó como el área $\mathscr{H}(x, x+4)$

b) Estimar la población media $\left(\boldsymbol{P}_{m}\right)$ correspondiente al período $(z, z+5)$ para el grupo de edad $(x, x+4)$ :

$$
P_{m}=\frac{P_{z}+P_{z+5}}{2}
$$

en donde: $P_{z}=$ población al $1^{\circ}$ de enero del año $z$

$P_{z+5}=$ población al $1^{\circ}$ de enero del año $z+5$

Gráfica 1

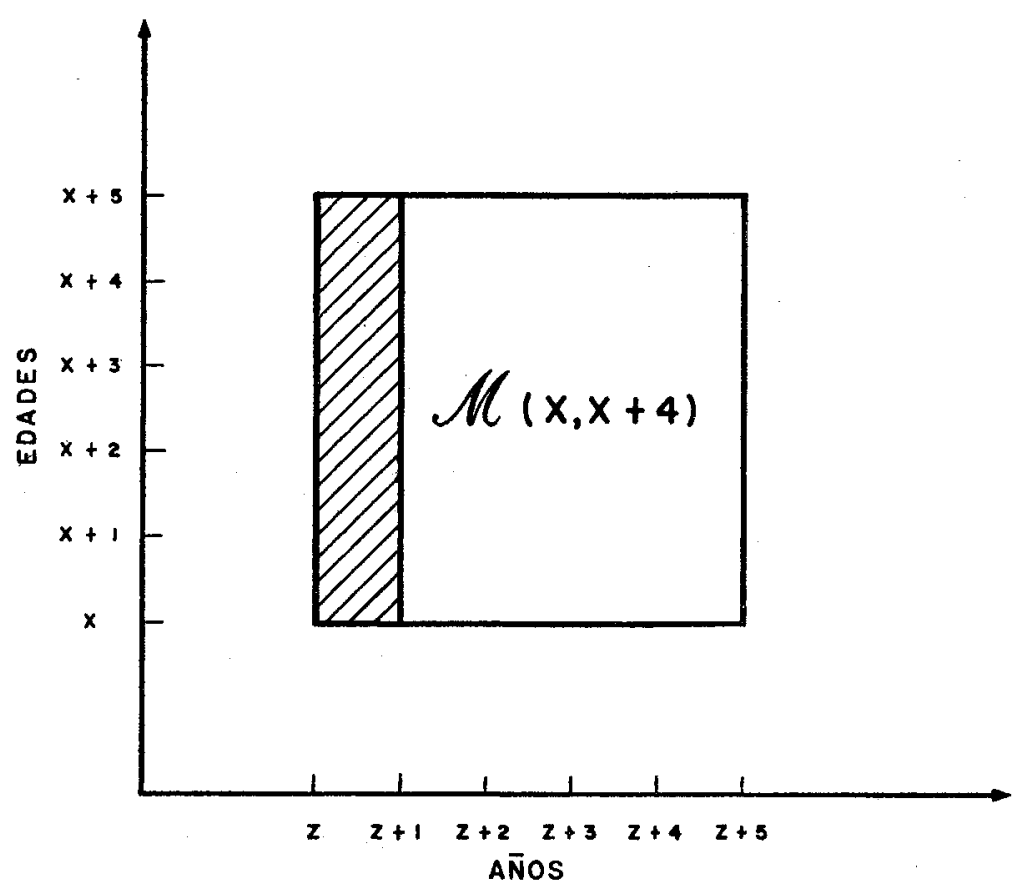


Los efectivos correspondientes a las poblaciones al $1^{\circ}$ de enero de los años censales se estimaron trasladando las poblaciones corregidas del 30 de junio de los años censales, ${ }^{8}$ al $1^{\text {o }}$ de enero de esos mismos años, mediante la tasa de crecimiento anual. Por otra parte, la estima-

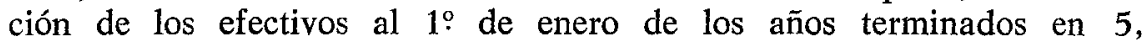
$\left(P_{z+5}\right)$, se obtienen a través de la media entre las poblaciones al $1^{\circ}$ de enero de los años censales que los enmarcan.

c) De esta manera la tasa de nupcialidad de segunda categoría $(T)$, por grupo de edades, es igual a

$$
T=\frac{\mathscr{U}(x, x+4)}{\frac{P_{z}+P_{z+5}}{2}}
$$

El conjunto de tasas de segunda categoría de nupcialidad civil que pudieron ser calculadas figuran en el cuadro 2 .

\section{2) Configuración de las tasas de nupcialidad por generaciones en un diagrama de Lexis}

El hecho de que en las estadísticas de Registro Civil no exista una clasificación de los matrimonios por edad y generación a que pertenecen los contrayentes, obligó a asimilar las tasas calculadas (tasas quinquenales por grupos de edad en años cumplidos) a tasas quinquenales por grupos de edad y generaciones (véase la gráfica 2).

Esta asimilación tuvo por finalidad poder llegar a construir "cuadros de nupcialidad por generaciones" que sirvieran de base para calcular las medidas que se utilizarán en la caracterización de la nupcialidad en cada grupo de generaciones y. sus variaciones a través del tiempo.

La gráfica 2 ilustra la forma en que se asimilaron las tasas de segunda categoría a tasas por grupo de generaciones y grupos de edad con el fin de posibilitar el análisis longitudinal. Las tasas de segunda categoría fueron dispuestas de tal modo que siguieran, en promedio, a las generaciones compuestas por personas que nacieron entre el $1^{\text {\% }}$ de julio del año $z+2.5$ y el 30 de junio del año $z+7.5$. Para facilitar la denominación de los grupos de generaciones obtenidos de esta manera se designaron con el año correspondiente a la generación central del grupo.

El ejemplo de la gráfica 2 ilustra la forma en que se determinó el grupo de generaciones nacidas alrededor de 1915: la tasa de segunda

8 G. Cabrera y R. Benítez Z., Tablas abreviadas de mortalidad, México, E1 Colegio de México, 1967 pp. 31-34. 
Cuadro 2

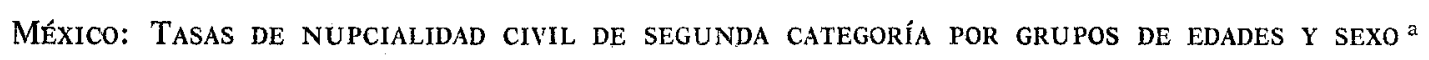

\begin{tabular}{|c|c|c|c|c|c|c|c|c|c|c|}
\hline \multirow{2}{*}{$\begin{array}{l}\text { Grapos } \\
\text { de odad }\end{array}$} & \multicolumn{10}{|c|}{ 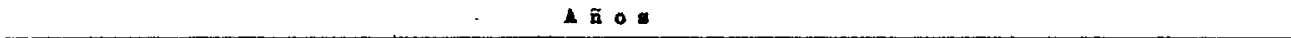 } \\
\hline & $\begin{array}{l}1922-\bar{b} \\
1924\end{array}$ & $\begin{array}{l}1925- \\
1927\end{array}$ & $\begin{array}{l}1930- \\
1934\end{array}$ & $\begin{array}{l}1935- \\
1939\end{array}$ & $\begin{array}{l}1940- \\
1944\end{array}$ & $\begin{array}{l}1945- \\
1949\end{array}$ & $\begin{array}{l}1950- \\
1954\end{array}$ & $\begin{array}{l}1955- \\
1959\end{array}$ & $\begin{array}{l}1960- \\
1964\end{array}$ & $\begin{array}{l}1965- \\
1969\end{array}$ \\
\hline \multicolumn{11}{|c|}{ Bombros } \\
\hline $15-19$ & 0.054 & 0.068 & 0.086 & 0.095 & 0.098 & 0.065 & 0.102 & 0.102 & 0.097 & 0.101 \\
\hline $20-24$ & 0.187 & 0.230 & 0.293 & 0.336 & 0.367 & 0.326 & 0.333 & 0.336 & 0.331 & 0.360 \\
\hline $25-29$ & 0.100 & 0.121 & 0.165 & 0.207 & 0.206 & 0.220 & 0.199 & 0.201 & 0.209 & 0.240 \\
\hline $30-34$ & 0.058 & 0.065 & 0.079 & 0.094 & 0.111 & 0.098 & 0.106 & 0.105 & 0.101 & 0.113 \\
\hline $35-39$ & 0.038 & 0.044 & 0.056 & 0.067 & 0.075 & 0.067 & 0.061 & 0.073 & 0.069 & 0.070 \\
\hline $40-49$ & 0.022 & 0.027 & 0.035 & 0.038 & 0.059 & 0.040 & 0.041 & 0.044 & 0.044 & 0.048 \\
\hline \multicolumn{11}{|c|}{ Yujeres } \\
\hline $15-19$ & 0.201 & 0.241 & 0.298 & 0.354 & 0.363 & 0.309 & 0.329 & 0.327 & 0.305 & 0.299 \\
\hline $20-24$ & 0.107 & 0.131 & 0.186 & 0.214 & 0.217 & 0.200 & 0.213 & 0.226 & 0.235 & 0.261 \\
\hline $25-29$ & 0.036 & 0.046 & 0.071 & 0.084 & 0.087 & 0.079 & 0.081 & 0.094 & 0.099 & 0.118 \\
\hline $30-34$ & 0.021 & 0.025 & 0.036 & 0.041 & 0.050 & 0.043 & 0.042 & 0.048 & 0.053 & 0.062 \\
\hline $35-39$ & 0.014 & 0.017 & 0.027 & 0.030 & 0.041 & 0.033 & 0.033 & 0.039 & 0.041 & 0.046 \\
\hline $40-49$ & 0.007 & 0.009 & 0.014 & 0.016 & 0.024 & 0.020 & 0.022 & 0.026 & 0.026 & 0.030 \\
\hline
\end{tabular}

Fuente: Anuarios Estadísticos y Censos de población 1921 y 1970 ; Poblaciones al $1^{0}$ de enero de los años 1930, 1940, 1950 y 1960: estimadas a partir de las "Tablas abreviadas de mortalidad", p. 31-34, G. Ca-

a Tasas calculadas para el total de matrimonios sin distinción del orden de unión.

b Para los años 1922-1924 y 1925-1927 se le dio a la tasa una dimensión quinquenal. 


\section{Gráfica 2}

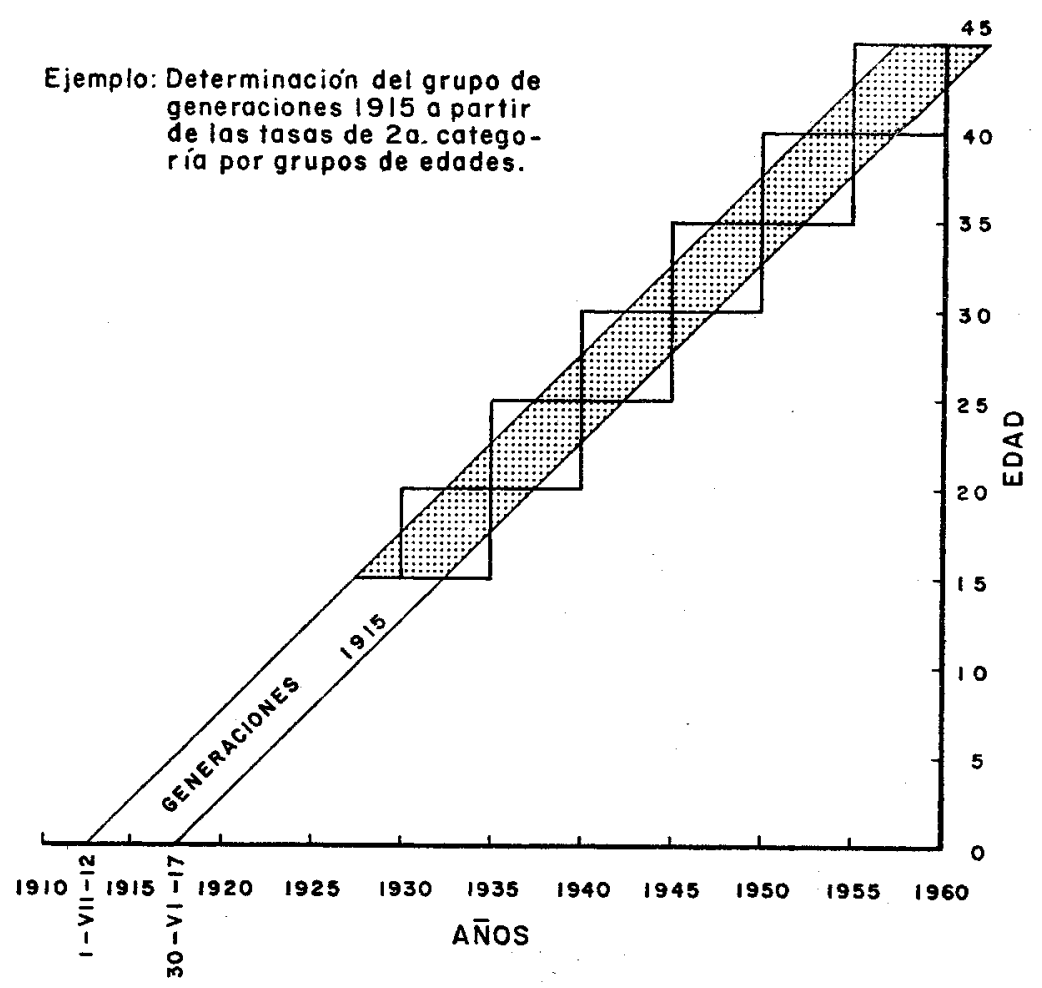

categoría de 15-19 años para los años 1930-1934 fue atribuida a las personas de la generación de 1915 que tuvieron esas mismas edades entre el $1^{\circ}$ de julio de 1927 y el 30 de junio de 1937; la tasa de segunda categoría de 20-24 años para los años 1935-1939 se aplicó a las personas de las generaciones de 1915 que tuvieron esas mismas edades entre el 19 de julio de 1932 y el 30 de junio de 1942; y así sucesivamente.

La suma de estas tasas hasta la edad exacta $x$ proporciona el número acumulado de matrimonios que figura en el "cuadro sobre nupcialidad de las generaciones de 1915" (véase el cuadro 3).

Fiste procedimiento fue aplicado a todas las generaciones completas, o sea, a todas aquellas cuya historia matrimonial pudo ser seguida entre las edades de 15 a 49 años cumplidos.

La adopción de 15 años como edad mínima al matrimonio fue impuesta por la forma en que la Dirección General de Estadística publica los datos relativos en las edades al matrimonio. La fijación de la edad máxima para contraer matrimonio, a los 49 años cumplidos, se justifica por el escaso número de matrimonios que se celebran después de esa edad. 
Cuadro 3

MÉxico: Tasa de NUPCialidad civil de SEgunda categoría, POR SEXO Y POR GENERACIONES

\begin{tabular}{|c|c|c|c|c|c|c|}
\hline \multirow{2}{*}{ Genoraoionen } & \multicolumn{6}{|c|}{ Grupes de edades $(x, x+4)$} \\
\hline & $15-19$ & $20-24$ & $25-29$ & $30-34$ & $35-39$ & $40-49$ \\
\hline $\begin{array}{c}1905 \\
M(x, x+4) \\
\text { Hombres } \\
\text { Mujereg } \\
\text { Matrinoniog seutul ados }\end{array}$ & $\begin{array}{r}54 \\
201\end{array}$ & $\begin{array}{l}230 \\
131\end{array}$ & $\begin{array}{r}165 \\
71\end{array}$ & $\begin{array}{l}94 \\
41\end{array}$ & $\begin{array}{l}75 \\
41\end{array}$ & $\begin{array}{l}40 \\
20\end{array}$ \\
\hline $\begin{array}{l}\text { Hombers } \\
\text { Mujores }\end{array}$ & 201 & $\begin{array}{l}284 \\
332\end{array}$ & $\begin{array}{l}449 \\
403\end{array}$ & $\begin{array}{l}543 \\
444\end{array}$ & $\begin{array}{l}618 \\
485\end{array}$ & $\begin{array}{l}698 \\
525\end{array}$ \\
\hline $\begin{array}{c}1910 \\
u\left(x_{,}, x+4\right) \\
\text { Hombses } \\
\text { Mujeros } \\
\text { Matrimon } 100 \text { 20umulation }\end{array}$ & $\begin{array}{r}68 \\
241\end{array}$ & $\begin{array}{l}293 \\
186\end{array}$ & $\begin{array}{r}207 \\
84\end{array}$ & $\begin{array}{r}111 \\
50\end{array}$ & $\begin{array}{l}67 \\
33\end{array}$ & $\begin{array}{l}41 \\
22\end{array}$ \\
\hline $\begin{array}{l}\text { Hombres } \\
\text { Xujeres }\end{array}$ & $\begin{array}{r}68 \\
241\end{array}$ & $\begin{array}{l}361 \\
427\end{array}$ & $\begin{array}{l}568 \\
511\end{array}$ & $\begin{array}{l}679 \\
561\end{array}$ & $\begin{array}{l}746 \\
594\end{array}$ & $\begin{array}{l}828 \\
638\end{array}$ \\
\hline 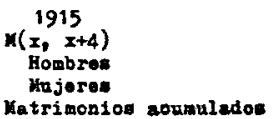 & $\begin{array}{r}86 \\
298\end{array}$ & $\begin{array}{l}336 \\
214\end{array}$ & $\begin{array}{r}206 \\
87\end{array}$ & $\begin{array}{l}98 \\
43\end{array}$ & $\begin{array}{l}61 \\
33\end{array}$ & $\begin{array}{l}44 \\
26\end{array}$ \\
\hline $\begin{array}{l}\text { Kombres } \\
\text { Ku jores }\end{array}$ & $\begin{array}{r}86 \\
298\end{array}$ & $\begin{array}{l}422 \\
512\end{array}$ & $\begin{array}{l}628 \\
599\end{array}$ & $\begin{array}{l}726 \\
642\end{array}$ & $\begin{array}{l}787 \\
675\end{array}$ & $\begin{array}{l}875 \\
727\end{array}$ \\
\hline $\begin{array}{c}1920 \\
\text { M(x, } x+4) \\
\text { Hombres } \\
\text { Mujeres } \\
\text { Xatrisonios aounulados }\end{array}$ & $\begin{array}{r}95 \\
354\end{array}$ & $\begin{array}{l}367 \\
217\end{array}$ & $\begin{array}{r}220 \\
79\end{array}$ & $\begin{array}{r}106 \\
42\end{array}$ & $\begin{array}{l}73 \\
39\end{array}$ & $\begin{array}{l}44 \\
26\end{array}$ \\
\hline $\begin{array}{l}\text { Hombros } \\
\text { Xujoros }\end{array}$ & $\begin{array}{r}95 \\
354\end{array}$ & $\begin{array}{l}462 \\
571\end{array}$ & $\begin{array}{l}682 \\
650\end{array}$ & $\begin{array}{l}788 \\
692\end{array}$ & $\begin{array}{l}861 \\
731\end{array}$ & $\begin{array}{l}949 \\
783\end{array}$ \\
\hline 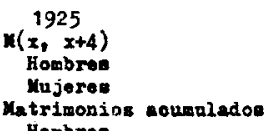 & $\begin{array}{r}98 \\
363\end{array}$ & 200 & $\begin{array}{r}199 \\
89\end{array}$ & $\begin{array}{r}105 \\
48 \\
\end{array}$ & $\begin{array}{r}69 \\
41\end{array}$ & $\begin{array}{r}48 \\
30 \\
\end{array}$ \\
\hline $\begin{array}{l}\text { Hombros } \\
\text { Hujeres }\end{array}$ & $\begin{array}{r}98 \\
363\end{array}$ & $\begin{array}{l}424 \\
563\end{array}$ & $\begin{array}{l}623 \\
644\end{array}$ & $\begin{array}{l}728 \\
692\end{array}$ & $\begin{array}{l}797 \\
733\end{array}$ & $\begin{array}{l}893 \\
793\end{array}$ \\
\hline 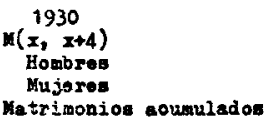 & $\begin{array}{r}65 \\
309\end{array}$ & $\begin{array}{l}333 \\
213\end{array}$ & $\begin{array}{r}201 \\
94\end{array}$ & $\begin{array}{r}101 \\
53\end{array}$ & $\begin{array}{l}70 \\
46\end{array}$ & $40^{40}$ \\
\hline $\begin{array}{l}\text { Hombroa } \\
\text { Mujorea }\end{array}$ & $\begin{array}{r}65 \\
309\end{array}$ & $\begin{array}{l}398 \\
522\end{array}$ & $\begin{array}{l}599 \\
616\end{array}$ & $\begin{array}{l}700 \\
669\end{array}$ & $\begin{array}{l}770 \\
715\end{array}$ & $\begin{array}{l}866 \\
775\end{array}$ \\
\hline
\end{tabular}

a Esta tasa fue estimada a partir de la tasa de la generación 1925 correspondiente a la misma edad.

\section{ANÁLISIS DE LOS "CUADROS DE NUPCIALIDAD CIVIL"}

Los cuadros de nupcialidad civil pudieron ser obtenidos para seis grupos de generaciones que abarcan la historia matrimonial legal completa de las personas nacidas entre mediados de 1902 y mediados de 1932.

Estos cuadros sirvieron de base para calcular las dos medidas de resumen que se utilizaron para trazar la evolución de la nupcialidad en México a través del tiempo: 
a) edad promedio a los matrimonios civiles, y

b) intensidad de los matrimonios civiles.

1. Arálisis de la edad promedio a la celebración de los matrimonos

La distribución estadística del total de matrimonios celebrados en una generación o cohorte en función de la edad, constituye el "calendario" de estos acontecimientos. En el caso de la mortalidad, el calendario viene a ser la distribución de las muertes desde el nacimiento hasta la desaparición de la generación. Por las características de la nupcialidad, el calendario es la repartición de las cantidades $m(x, x+a)$ correspondiente a los matrimonios celebrados entre los $15 \mathrm{y}$ los 50 años de edad, en que se supone, se han casado ya todos aquellos que lo harán por lo menos una vez. Este concepto puede ser aplicado transversalmente si se trabaja con datos del momento o longitudinalmente si los datos corresponden a generaciones o cohortes.

El calendario de un fenómeno demográfico puede ser resumido a través de la edad media a la cual se produce dicho fenómeno. La edad media cuyo cálculo se detalla a continuación representa, en el cáso de este trabajo, la edad a la celebración del matrimonio civil, cualquiera que sea el orden de la unión contraída en un grupo de generaciones.

La fórmula utilizada en el cálculo de la edad media fue la siguiente (véase el cuadro 3):

$$
m=\frac{\sum_{x=15}^{45} m(x, x+4) \cdot(x+2.5)}{\sum_{x=15}^{45} m(x, x+4)}
$$

en donde: $(x+2.5)$ es la edad media a la cual se celebran los matrimonios del grupo de edad $(x, x+4)$; y $m(x, x+4)$ el número de matrimonios civiles celebrados entre las edades cumplidas, $x$ y $(x+4)$ (véase el cuadro 4).

En el cuadro 4, donde figuran las edades medias al matrimonio de los seis grupos de generaciones completas resalta el elevado nivel de estas edades. Generalmente, en los países que tienen una fecundidad alta como es el caso de México, la edad media al matrimonio es baja, de allí que parezcan sorprendentes las edades medias obtenidas.

En realidad, el cálculo efectuado corresponde al de la edad media en el momento de la inscripción civil de los matrimonios y no a la edad promedio a la formación de las uniones. La diferencia entre éstos dos conceptos surge de que el registro de un matrimonio civil puede traducir dos fenómenos distintos cuya influencia puede ser esquematizada de la manera siguiente: 
a) Caso 1: matrimonio civil de dos personas solteras a una edad $x$.

b) Caso 2: a la edad $\mathrm{x}$ se forma una unión libre o religiosa que viene a legalizarse $t$ años más tarde bajo el efecto de presiones de distinto tipo (v. gr., la necesidad del certificado de matrimonio para obtener ciertos derechos).

\section{Cuadro 4}

MÉxico: EdAd promedio al MATRIMONIO E INTENSIDAd DEL MATRIMONIO CIVIL A LOS 50 AÑOS, POR GRUPO DE GENERACIONES, 1905-1930

\begin{tabular}{|c|c|c|c|c|c|}
\hline \multirow{2}{*}{ Generaoiones } & \multicolumn{3}{|c|}{ Edad media } & \multicolumn{2}{|c|}{ Intensidades } \\
\hline & Hombrea & - & Mujerea & Hombres & kujeres \\
\hline 1905 & 28.82 & & 24.93 & 698 & 525 \\
\hline 1910 & 28.00 & & 24.37 & 828 & 638 \\
\hline 1915 & 27.60 & & 23.92 & 875 & 727 \\
\hline 1920 & 27.51 & & 23.50 & 949 & 783 \\
\hline 1925 & 27.81 & & 23.80 & 893 & 793 \\
\hline 1930 & 28.15 & & 24.41 & 866 & 775 \\
\hline
\end{tabular}

a Por mil.

En los dos casos, la edad $x$ es la edad real que debe tomarse como tal a la formación de la unión, pero la edad al matrimonio civil es $x$ en el caso 1 y $(x+t)$ en el caso 2 .

Entre mayor sea el número de matrimonios civiles del tipo $b$ en una población, mayor será la diferencia también entre la edad promedio a la formación de las uniones y la edad promedio al inatrimonio civil.

De esta manera, cualquier interpretación que se haga en función de la edad media al matrimonio (v. gr., el estudio de las variaciones del número promedio de hijos nacidos vivos), tendrá que considerar el efecto perturbador de la legalización tardía de uniones.

Otro efecto perturbador importante, que redunda también en una elevación de la edad promedio al matrimonio civil, se produce por los matrimonios de segundo o mayor orden cuya magnitud y distribución por edad se desconoce. Mientras las estadísticas del Registro Civil no clasifiquen los matrimonios según el estado civil anterior de los contrayentes, no se podrá afinar más el análisis de la nupcialidad. Un recurso para resolver este problema sería recurrir a las encuestas demográficas, a través de las cuales se pueden reconstruir las historias demográficas completas de los individuos interrogados. $\mathrm{El}$ inconveniente de este método es que no permite captar las variaciones del fenómeno en lapsos más o menos largos, salvo que las encuestas se efectúen periódicamente.

En el mismo cuadro 4 vemos que las edades promedio tienden a disminuir entre las generaciones de 1905 y 1920 para luego aumentar en las generaciones posteriores.

Esta evolución puede ser seguida en forma más precisa a través del cuadro 5. En este cuadro se enmarcaron los elementos del calendario correspondientes a las edades que atraviesan el período 1930-1940 el cual 
Cuadro 5

MÉxico: Calendario de los Matrimonios Civiles EN LAS generaciones OBSERVADAS, 1905-1930

\begin{tabular}{|c|c|c|c|c|c|c|}
\hline \multirow{2}{*}{$\begin{array}{l}\text { Gropos } \\
\text { do oded }\end{array}$} & \multicolumn{6}{|c|}{ Coneraelonen } \\
\hline & 1905 & 1910 & 1915 & 1920 & 1925 & 1930 \\
\hline \multicolumn{7}{|c|}{ Honbres } \\
\hline $15-19$ & 8 & 8 & 10 & 10 & 11 & 8 \\
\hline $20-24$ & 33 & 35 & 41 & 39 & 36 & 38 \\
\hline $25-29$ & 24 & 25 & 23 & 23 & 22 & 23 \\
\hline $30-34$ & 13 & 13 & 11 & 11 & 12 & 12 \\
\hline $35-39$ & 11 & 8 & 7 & 8 & 8 & 8 \\
\hline $40-49$ & 11 & 10 & 10 & 9 & 11 & 11 \\
\hline \multicolumn{7}{|c|}{ Enitere: } \\
\hline $15-19$ & 38 & 38 & 41 & 45 & 46 & 40 \\
\hline $20-24$ & 25 & 29 & 29 & 28 & 25 & 27 \\
\hline $25-29$ & 13 & 13 & 12 & 10 & 10 & 12 \\
\hline $30-34$ & 8 & 8 & 6 & 5 & 6 & 7 \\
\hline $35-39$ & $8)$ & 5 & 4 & 5 & 5 & 6 \\
\hline $40-49$ & 8 & 7 & 1 & 7 & 8 & 8 \\
\hline
\end{tabular}

Nota: Las cifras enmarcadas corresponden a valores del calendario de las generaciones observadas para el período 1930-1939.

se caracterizó por un aumento sustancial de la nupcialidad civil. ${ }^{9}$ Si se observa detenidamente el cuadro se constata que los valores más altos del calendario, enmarcados en el cuadro, corresponden para un mismo grupo de edades en cada generación a los afectados en el período de alza de la nupcialidad. Como este efecto se da en las generaciones para edades cada vez más jóvenes, especialmente entre las generaciones de 1905 y 1920, puede ser ligado al descenso que sufrió la edad promedio en el lapso transcurrido entre esos mismos dos grupos de generaciones.

Si se acepta que la nupcialidad civil aumentó rápidamente en el período 1930-1940, debido en gran parte a la legalización de uniones, puede hacerse el razonamiento siguiente: el calendario de los matrimonios de las generaciones de 1905 y 1920 se encuentra compuesto por un calendario básico que varía poco entre esas generaciones pero que fue deformado en forma selectiva por las legalizaciones de uniones en función "de su paso" a través del período 1930-1940. Una vez concluído este período de perturbaciones, el calendario volvió a asemejarse al calendario básico.

El único elemento del cual se dispone para apoyar esta hipótesis son los valores del calendario de los matrimonios civiles que no fueron afectados por el período perturbado 1930-1940 y que como se puede apreciar en el cuadro 5 (valores no enmarcados) permanecieron relativamente constantes de una generación a otra.

9 J. Quilodrán, loc. cit. 
Por otra parte, se observa que la edad media después de un período de variación más o menos amplio, alcanza valores muy similares en las generaciones que fueron poco afectadas por la perturbación (generaciones de 1905 y 1930). En el caso de los hombres, la edad promedio al matrimonio en la generación de 1905 fue 28.81 años y 28.15 en la generación de 1930; en el caso de las mujeres, las cifras fueron de 24.93 y 24.41 respectivamente.

\section{Evolución de las intensidades en las generaciones}

La acumulación de los valores que corresponden a la serie de acontecimientos proporciona la estimación de la intensidad pura del fenómeno. La acumulación de los valores de la serie de matrimonios civiles $m(x, x+4)$ hasta los 50 años (véase el cuadro 4), proporciona una estimación de la intensidad de la nupcialidad civil para un efectivo de 1000 hombres o mujeres en cada grupo de generaciones.

Los valores de las intensidades contenidos en el cuadro 4 se elevan en forma contínua para los hombres entre la generación 1905 y 1920 y para las mujeres de la generación de 1905 hasta la generación de 1925, mientras que para las generaciones posteriores se vislumbra cierta estabilización. Si se comparan los valores de las generaciones extremas, 1905 y 1930 , el incremento de la intensidad del matrimonio civil fue de $19.4 \%$ en el caso de los hombres y de $32.3 \%$ en el caso de las mujeres. Cabe señalar que la intensidad del matrimonio civil ha sido siempre mayor para los hombres, aún cuando la relación entre la intensidad mujer/hombre pasó de 0.75 , en las generaciones de 1905, a 0.89 en las generaciones 1930.

Este fuerte incremento en la intensidad de los matrimonios civiles sería un primer rasgo importante, por lo que sería conveniente analizar el valor inusitadamente elevado de la intensidad correspondiente a la generación masculina de 1920.

Dichos aumentos pueden explicarse por ciertos desequilibrios en las poblaciones casaderas producidos por una recuperación de la natalidad en México alrededor de los años veinte, que corresponden a los últimos años de la Revolución. Aún cuando no se cuenta con análisis detallados respecto a este aumento en los nacimientos, es lógico pensar que se dio, por el simple hecho de la celebración de uniones postergadas y la reunión de parejas cuya separación había retardado el nacimiento de algunos de sus hijos.

La relación entre el incremento de los nacimientos y el aumento de la intensidad al matrimonio en las generaciones nacidas durante ese período puede explicarse en la siguiente forma:

Teniendo en consideración que las generaciones femeninas contraen matrimonio con hombres de generaciones más viejas, especialmente con las del grupo inmediato superior, cuando las mujeres pertenecientes a 
las generaciones nacidas alrededor de 1920 llegaron a la edad de contraer matrimonio, encontraron que las generaciones masculinas con quienes les correspondía casarse, o sea, las generaciones de 1915, nacidas justo antes de la recuperación de la natalidad, eran, por este mismo hecho, menos numerosas.

Este desequilibrio en la población "casadera" parece haber sido resuelto a través de una mayor intensidad de la nupcialidad en las generaciones masculinas de 1915 y 1920 . Es decir, las mujeres de las generaciones de 1920 se habrían casado no sólo con hombres de la generación que les correspondía (1915) sino también en una mayor proporción que la acostumbrada, con hombres de su misma generación.

La afirmación no puede ser tajante porque no hay elementos para disociar el efecto combinado del alza normal de la nupcialidad civil que se observa desde 1930 y del desequilibrio de la población casadera de las generaciones afectadas por la recuperación de la natalidad.

\section{Conclusiones}

Del análisis de la nupcialidad en función de la edad promedio al matrimonio civil y de la intensidad de este mismo a través de las generaciones, se desprenden dos tendencias en forma bastante clara:

a) Un ascenso marcado de la intensidad entre las generaciones 1905 y $1930, y$

b) la existencia de una edad promedio que no revela modificaciones realmente profundas a pesar de las variaciones que presenta durante el período estudiado.

Podría pensarse que el incremento de los matrimonios civiles, que refleja el alza de la intensidad del matrimonio en las generaciones, produjera cambios significativos en la edad promedio al matrimonio civil. Es decir, que el patrón de edad al matrimonio civil fuera distinto al de los otros tipos de unión y que por lo tanto, el hecho de que un número cada vez mayor de parejas contrajera una unión legal, haría variar en forma importante la edad promedio al matrimonio civil. Es muy probable que esto no ocurriera debido a:

a) las características de los datos utilizados en el cálculo de la edad promedio al matrimonio civil. Como se sabe, esta edad no corresponde a la edad real a la formación de las uniones civiles pues comporta el efecto de las nupcias de segundo o mayor orden y de las legalizaciones de uniones cuyas distribuciones por edad se desconoce. De ahí que, cualquier desplazamiento en el calendario de estas dos variables pudo haber contrarrestado y ocultado el efecto de un cambio de comportamiento en relación con la edad al matrimonio civil. 
b) que efectivamente la edad promedio al matrimonio civil no hubiera cambiado con el incremento de éste. Esto pudo haber ocurrido si las parejas, que en forma cada vez más numerosa contraían una unión de tipo legal, hubieran tenido un comportamiento respecto a la edad al casarse similar al de aquellas que contraían matrimonio civil independientemente del monto del aumento que éste estuviera sufriendo.

Si como todo parece indicar, el alza de los matrimonios civiles durante el período estudiado, se efectuó en detrimento del matrimonio religioso principalmente,,$^{10}$ esta segunda hipótesis parece bastante plausible. Aun cuando se desconoce la edad media a este tipo de unión, puede presumirse que debe ser semejante a la del matrimonio civil, pues una vez adoptada la decisión de contraer matrimonio que es el hecho que determina la edad a la formación de la unión, la busqueda de una sanción religiosa o civil, o ambas, obedece exclusivamente al deseo de legitimarlas.

Para confirmar las tendencias anteriores, sería preciso disponer de series estadísticas más completas, tanto por lo que se refiere al número de años como a la cantidad de datos sobre el mismo fenómeno. Una vez cumplida esta condición sería factible realizar una interpretación de las variaciones de la nupcialidad y sus consecuencias.

A pesar de las limitaciones que puede presentar la aplicación de un enfoque longitudinal con datos no adecuados a un estudio por generaciones, pareció interesante mostrar las posibilidades concretas que un análisis de este tipo puede ofrecer.

La utilización del enfoque longitudinal era indispensable para obtener las tendencias a largo plazo, sobre todo tratándose de. un fenómeno demográfico como la nupcialidad, tan expuesta a fluctuaciones de tipo conyuntural.

\section{APÉNDICE METODOLÓGICO ${ }^{1}$}

En una generación cualquiera, $\mathscr{H}_{x}$ representa el número de matrimonios observados a la edad $x$ en años cumplidos y $P_{x}$ la población presente a la edad exacta $x$.

En ausencia de mortalidad, el número de matrimonios en la generación observada sería igual a $\mathscr{M}_{x}+e_{x}$ donde $e_{x}$ constituye el número de matrimonios impedidos por la mortalidad.

En ausencia de mortalidad, la población $P_{x}$ se mantiene constante, y el número medio de matrimonios de la generación a la edad $x$ viene a ser igual a:

$$
m_{x}=\frac{\mathscr{M} x+e_{x}}{P_{x}}
$$

10 J. Quilodrán, ibid.

1 Adaptado de L. Henry: Demographie, analyse et modèles, París, Larousse, 1972, p. 96. 
Este número medio de matrimonios $\left(m_{x}\right)$ representa al mismo tiempo la probabilidad, para los individuos de edad exacta $x$, de contraer matrimonio a la edad $x$ en años cumplidos. En el caso de acontecimientos renovables, el cociente de nupcialidad es igual al número medio de matrimonios a una edad dada.

Ahora, si $q_{x}$ representa la probabilidad de morirse a la edad $x, p_{x} m_{x} q_{x}$ individuos se casarán ${ }^{2}$ y morirán durante el año $x$.

Si consideramos que los individuos que se casaron y que van a morirse antes de la edad $x+1$ están incluídos en el número de matrimonios observados, $\mathscr{H}_{x}$, faltaría estimar el número de individuos que deberían haberse casado a la edad $x$ pero que no lo hicieron por haberse muerto antes de haber podido casarse, $e_{x}$. No existe razón alguna para pensar que estos últimos efectivos deban ser diferentes de aquellos que se casaron antes de morirse, por lo tanto, $e_{x}$, puede ser estimado a través d la fórmula siguiente:

$$
e_{x}=\frac{P_{x} m_{x} q_{x}}{2}
$$

en donde: $\boldsymbol{P}_{x} q_{x}=d_{x}$

a su vez, $d_{x}$ representa el número total de defunciones ocurridas entre las edades $x$ y $(x+1)$.

Reemplazando estos valores en la fórmula, 1) tendremos que:

$$
m_{x}=\frac{\mathscr{A} x}{P_{x}-\frac{d_{x}}{2}}
$$

E1 denominador de 2) constituye la población promedio a la edad $x$, $\left(P m_{x}\right)$. De este modo, se llega a establecer el número medio de matrimonios por mujer $\left(m_{x}\right)$ :

$$
\frac{\mathscr{A} x}{P m}
$$

que por definición, es la tasa de segunda categoría correspondiente a la edad $x$.

El presente razonamiento es válido bajo la hipótesis de que:

a) la mortalidad no varía en función del estado civil para una misma edad $x$. Probablemente esta hipótesis no se cumple debido a la mortalidad diferencial que normalmente existe entre solteros y casados, pero puede ser aceptada en la medida en que el error al cual conduce es insignificante;

b) que el efecto perturbador de las migraciones internacionales es limitado dado el escaso volumen de éstas en el caso de México. En lo que respecta a las migraciones internas no afectan las tasas calculadas.

2 En el caso de independencia entre los fenómenos nupcialidad y mortalidad. 